\title{
Resonant motions of supermassive black hole triples
}

\author{
Wei Hao ${ }^{1}$, Rainer Spurzem ${ }^{2,3,4,1}$, Thorsten Naab ${ }^{1}$, Long Wang $^{4,5}$, \\ M. B. N. Kouwenhoven ${ }^{4}$, Pau Amaro-Seoane ${ }^{6}$ \\ and Rosemary A. Mardling ${ }^{7,8}$ \\ ${ }^{1}$ Max-Planck-Institut für Astrophysik, Karl-Schwarzschild-Str. 185741 Garching, Germany \\ email: elvis@mpa-garching.mpg.de \\ ${ }^{2}$ National Astronomical Observatories of China, Chinese Academy of Sciences, 20A Datun \\ Rd., Chaoyang District, 100012, Beijing, P.R. China \\ ${ }^{3}$ Astronomisches Rechen-Institut, Zentrum für Astronomie, Univ. of Heidelberg, \\ Mönchhof-Strasse 12-14, 69120 Heidelberg, Germany \\ ${ }^{4}$ Kavli Institute for Astronomy and Astrophysics, Peking University, Yi He Yuan Lu 5, \\ Haidian District, Beijing 100871, P.R. China \\ ${ }^{5}$ Department of Astronomy, School of Physics, Peking University, Yi He Yuan Lu 5, Haidian \\ District, Beijing 100871, P.R. China \\ ${ }^{6}$ Max Planck Institut für Gravitationsphysik (Albert-Einstein-Institut), D-14476 Potsdam, \\ Germany \\ ${ }^{7}$ School of Mathematical Sciences, Monash University, Victoria 3800, Australia \\ ${ }^{8}$ Astronomy Department of the University of Geneva, Geneva Observatory, $51 \mathrm{Ch}$. des \\ Maillettes, CH1290 Versoix, Switzerland
}

\begin{abstract}
Triple supermassive black holes (SMBH) can form during the hierarchical mergers of massive galaxies with an existing binary. Perturbations by a third black hole may accelerate the merging process of an inner binary, for example through the Kozai mechanism. We analyze the evolution of simulated hierarchical triple SMBHs in galactic centers, and find resonances in the evolution of the semi-major axis, the eccentricity and the inclination, for both the inner and the outer orbits of the triple system, which are not only Kozai like. Through resonant oscillations, SMBH can trigger a significant increase of the inner SMBH binary eccentricity shortening the merger timescale expected from gravitational wave (GW) emission. As hierarchical triple SMBHs may be frequent in massive galaxies, the influence of orbital resonances is of great importance to our understanding of black hole coalescence and gravitational wave detection. Although Kozai mechanism is believed to play an important role in this process, detailed studies on the pattern of these resonances is necessary.
\end{abstract}

Keywords. black hole physics, kinematics and dynamics, oscillations, etc.

\section{Introduction}

Supermassive black holes (SMBHs) are common in the centers of massive galaxies (e.g. Kormendy \& Ho 2013). Within the framework of a cold dark matter cosmology, these SMBHs would form SMBH binaries as a consequence of hierarchical mergers of their host galaxies White \& Rees 1978, Begelman, Blandford \& Rees 1980. Massive galaxies are typically the product of multiple mergers of galaxies (e.g. De Lucia et al. 2006). The long time span from 0.1 to 1 Gyr for the coalesce of SMBH binaries would largely increase the possibility of a secondary merger before the final coalesce of these SMBH binaries (Khan et al. 2013), if the merger timescale is much larger than the timescale between two mergers. Since most massive galaxies should have experienced multiple mergers during 

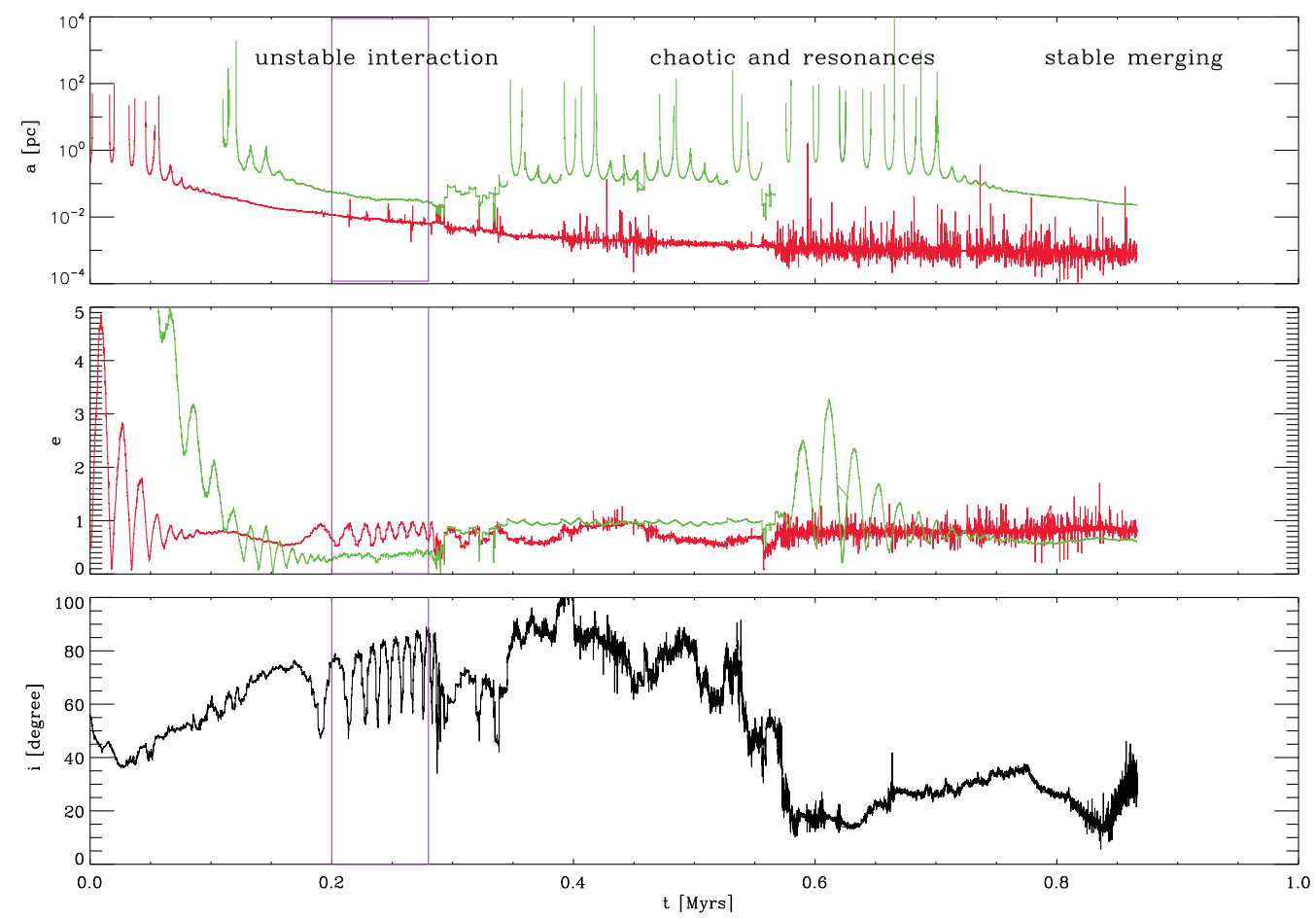

Figure 1. Full evolution of the semi-major axis, eccentricity, and relative inclination with time in this example simulation. This plot shows how semi-major axis $A$, eccentricity $E C C$ and inclination angle difference between inner and outer binary $i_{\text {in }}-i_{\text {out }}$ as a function of time. Red curve represents inner binary and green curve represents outer binary, and the same bellow. The discrete part of the semi-major evolution, especially those of the outer binary, suggesting the three body system is unbound sometimes, but they could be pulled back by the surround stars afterwards.

their lifetime, a triple or more SMBH system would frequently form. If we take the central inner SMBH binary as a whole respect to the third SMBH on wider orbit, we can define them as outer binary, and this inner binary together with outer binary is called a hierarchical triple system.

The impact of the third SMBH on the merging SMBH binary can be crucial, either by ejection of one component of the three or by perturbing the orbit of the inner binary (Valtonen, Mikkola \& Heinamaki 1994). Besides, repeated encounters between the third SMBH and the progenitor SMBH binary would possibly excite eccentricity of the binary (Makino \& Ebisuzaki 1994). For a hierarchical triple system, if the inclination angle between the orbital planes of inner and outer binary is higher than $39.2^{\circ}$, these binaries would oscillate with a Kozai resonance (Kozai 1962). This process could greatly excite the inner binary's eccentricity, which is important for the merging SMBH binary since the GW emission efficiency is a strongly depend on their semi-major axis and eccentricity (Peters 1964).

Although Kozai (-like) mechanism is believed to play a pivotal role during the evolution of hierarchical triple SMBHs in galaxy centers, it is not clear how exactly it could impact the merger of the inner binary in the environment of galactic nuclei. 

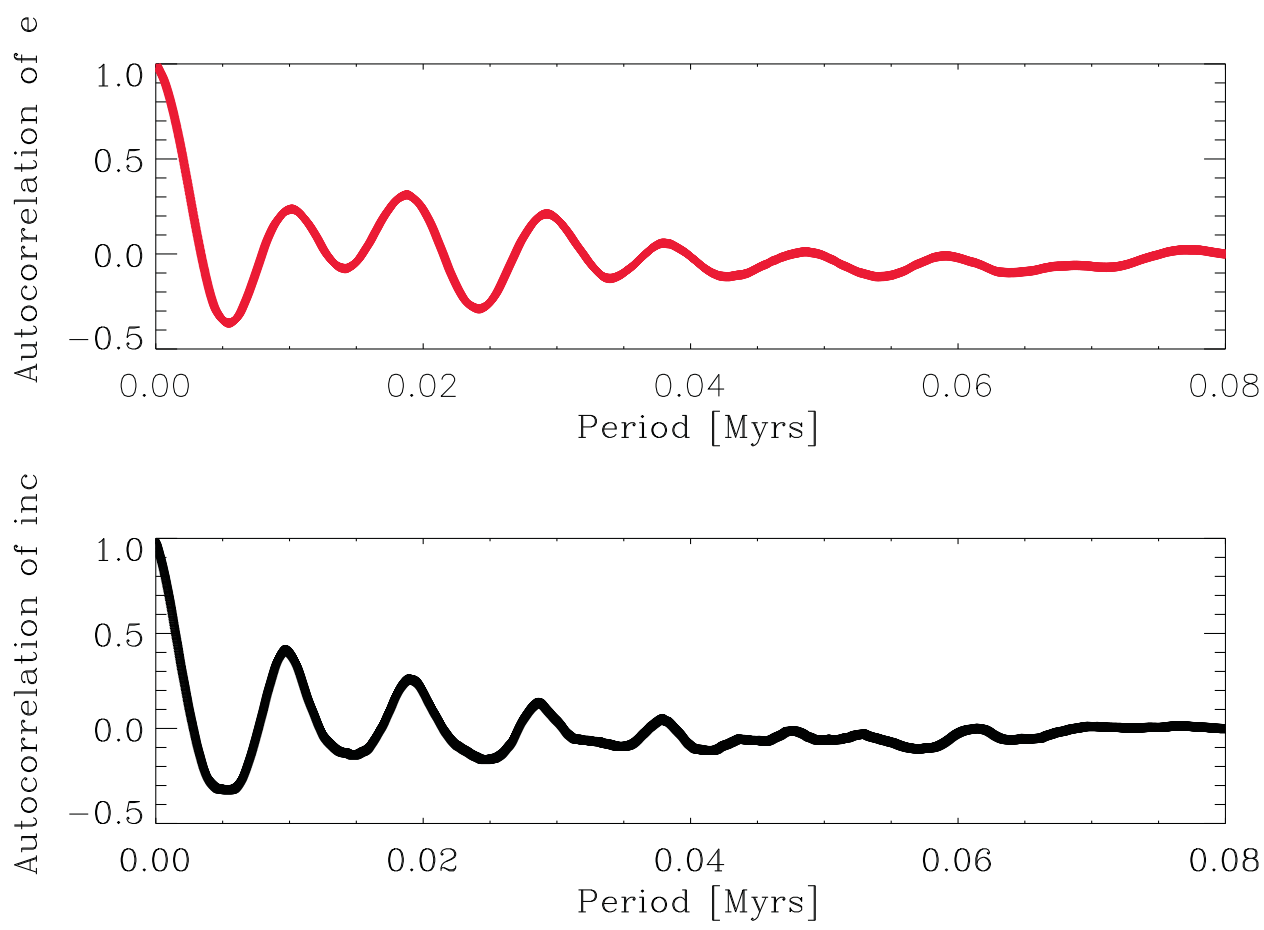

Figure 2. Auto-correlation result of inner eccentricity and inclination angle

\section{Simulation setup}

Our simulation has a total particle number of 64000 , including three SMBHs with 0.01 unit mass each and the 63997 surrounding equal mass star clusters with a total mass of 0.97 unit mass. All our particles are non-evolving point mass. Plummer model with $n=5$ polytrope is applied for the stellar system. We start the simulation from parsec scale, and terminate our simulation once the inner binary reach to a separation of milliparsec scale, which is the typical distance for triggering immediate merger by GW emission. We used a special version of NBODY6 to model this process (Amaro-Seoane et al. 2010 Aarseth, S., 2003).

\section{Results}

Figure 1 shows an example of Kozai-like resonance found in one of our simulation data. When looking at the range in the magenta box, we can see clear resonance between eccentricity of inner binary and the inclination angle. Figure 2 shows the result of autocorrelation for eccentricity of the inner binary and inclination angle, indicating it is oscillating with a fine period of $0.01 \mathrm{Myr}$, which is about a hundred times higher than standard Kozai timescale. This Kozai timescale is given by Kiseleva \& Chernin (1988)

$$
\tau=\frac{2 T_{\text {out }}^{2}}{3 \pi T_{\text {orb }}} \frac{m_{1}+m_{2}+m_{3}}{m_{3}}\left(1-e_{\text {out }}^{2}\right)^{3 / 2} .
$$

Although this oscillation is not a pure Kozai resonance, but it played the similar role on exciting the eccentricity of the inner binary. And this would accelerate the merger process. Besides, several other pattern of oscillations are found as well, (e.g., oscillations 
between outer eccentricity and inclination,) indicating more investigation should be apply to reveal the complexity of this hierarchical triple system embedded in the galaxy centers.

\section{References}

Aarseth, S., 2003, Gravitational N-body Simulations (Cambridge, Cambridge University Press, 2003, 430

Amaro-Seoane P., Sesana A., Hoffman L., Benacquista M., Eichhorn C., Makino J., \& Spurzem R., 2010, MNRAS, 402, 2308

Begelman M. C., Blandford R. D., \& Rees M. J., 1980, Nature, 287, 307

De Lucia G., Springel V., White S. D. M., Croton D., \& Kauffmann G., 2006, MNRAS, 366, 499

Khan F. M., Holley-Bockelmann K., Berczik P., \& Just A., 2013, ApJ, 773, 100

Kiseleva L. G. \& Chernin A. D. ., 1988, Soviet Astronomy Letters, 14, 412

Kormendy J. \& Ho L. C., 2013, ARAA, 51, 511

Kozai Y., 1962, AJ, 67, 591

Makino J. \& Ebisuzaki T., 1994, ApJ, 436, 607

Peters P. C., 1964, Physical Review, 136, 1224

Valtonen M. J., Mikkola S., \& Heinamaki P., 1994, American Astronomical Society Meeting Abstracts No.184, 26, 913

White S. D. M. \& Rees M. J., 1978, MNRAS, 183, 341 